\title{
Vibrational dynamics of ice in reverse micelles
}

\author{
Adriaan M. Dokter, ${ }^{\text {a) }}$ Christian Petersen, Sander Woutersen, and Huib J. Bakker \\ FOM Institute for Atomic and Molecular Physics, Kruislaan 407, 1098 SJ Amsterdam, The Netherlands
}

(Received 5 November 2007; accepted 27 November 2007; published online 31 January 2008)

\begin{abstract}
The ultrafast vibrational dynamics of $\mathrm{HDO}: \mathrm{D}_{2} \mathrm{O}$ ice at $180 \mathrm{~K}$ in anionic reverse micelles is studied by midinfrared femtosecond pump-probe spectroscopy. Solutions containing reverse micelles are cooled to low temperatures by a fast-freezing procedure. The heating dynamics of the micellar solutions is studied to characterize the micellar structure. Small reverse micelles with a water content up to approximately 150 water molecules contain an amorphous form of ice that shows remarkably different vibrational dynamics compared to bulk hexagonal ice. The micellar amorphous ice has a much longer vibrational lifetime than bulk hexagonal ice and micellar liquid water. The vibrational lifetime is observed to increase linearly from 0.7 to 4 ps with the resonance frequency ranging from 3100 to $3500 \mathrm{~cm}^{-1}$. From the pump dependence of the vibrational relaxation the homogeneous linewidth of the amorphous ice is determined $\left(55 \pm 5 \mathrm{~cm}^{-1}\right)$. (C) 2008 American Institute of Physics. [DOI: 10.1063/1.2826376]
\end{abstract}

\section{INTRODUCTION}

Reverse micelles have proven to be excellent model systems for studying the properties of water in strong confinement. ${ }^{1}$ Such micelles consist of small aqueous droplets that are coated by a layer of surfactant molecules and are dispersed in an apolar solvent. The hydrophilic head groups of the surfactant molecules point toward the micelle interior, while their hydrophobic tails point outward to the exterior solvent. For a large variety of surfactants and solvents, the size of the micelles increases monotonically with the molar ratio [water]/[surfactant], conventionally denoted by the parameter $w_{0}$. This property makes it very easy to vary the degree of confinement of water inside the micelles.

The confinement of liquid water to small volumes changes its structure and dynamics ${ }^{2-13}$ but also its phase behavior. Liquid water may be supercooled to much lower temperature when confined to micrometer sized capillaries. In the case of strongly confined ice, surface effects become important, and this may induce the formation of ice structures different from normal hexagonal ice $I h .^{14,15}$

In this work, reverse micellar solutions are cooled to low temperatures to study the effect of micellar confinement on the freezing behavior of water. Low temperature reverse micelles have become of relevance to the field of cryoenzymology and low temperature structural biology ${ }^{16,17}$ since these systems can be used to solubilize large amounts of protein. Several techniques have previously been used to study the freezing behavior of reverse micelles, including linear spectroscopy, ${ }^{18}$ fluorescent probes, ${ }^{19}$ and NMR. ${ }^{20}$ These studies show that freezing occurs within the water pool at temperatures between 210 and $265 \mathrm{~K}$, and that water inside reverse micelles may be supercooled to $240 \mathrm{~K} \cdot{ }^{19,20}$ Also, the hydrogen bond network behavior of the water pool exhibits a strong dependence on $w_{0}$ and does not approximate that of bulk water until $w_{0}=40{ }^{18}$

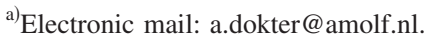

The sample preparation of stable reverse micelles at low temperatures is challenging. Breakdown of micellar structure was observed for slowly frozen reverse micellar solutions. ${ }^{19-22}$ Using a fast-freezing procedure we find that clear low temperature reverse micellar dispersions can be prepared, suggesting the retainment of the room-temperature micellar structure. Micelle stability is investigated by studying the heating dynamics of the micellar solutions. We find that the freezing leads to the formation of an amorphous type of ice in the interior of small reverse micelles. We study the ultrafast vibrational dynamics of this micellar amorphous ice and find that it differs markedly from that of bulk ice.

\section{EXPERIMENTAL}

\section{A. Sample preparation}

A solution of nanometer-sized water droplets forms when preparing an emulsion of water in an apolar solvent by addition of a surfactant. We prepare nanometer-sized ice crystals by rapidly freezing such a micellar solution. We used the anionic lipid surfactant sodium bis(2-ethylhexyl) sulfosuccinate (AOT), which is known to form reverse micelles that are reasonably monodisperse at room temperature $[\sim 15 \%$ size polydispersity (standard deviation size/mean size) $].^{23,24}$ The size of the water droplets can be easily varied by changing the molar water-to-AOT ratio, conventionally denoted by the parameter $w_{0}=[$ water $] /[\mathrm{AOT}]$.

The investigated samples were mixtures of the surfactant salt AOT (SigmaUltra grade $>99 \%$, Aldrich), iso-octane (anhydrous grade 99.8\%, Aldrich), pentane (anhydrous grade $>99 \%$, Aldrich), and water $\left(\mathrm{H}_{2} \mathrm{O}\right.$ HPLC grade, Aldrich. $\mathrm{D}_{2} \mathrm{O}>99.9 \%$, Apollo Scientific). We used isotopic dilutions of $\mathrm{HDO}$ in $\mathrm{D}_{2} \mathrm{O}\left(\mathrm{H}_{2} \mathrm{O}: \mathrm{D}_{2} \mathrm{O}=1: 40\right)$ to prevent the signals to be affected by intermolecular resonant energy transfer of the $\mathrm{OH}$-stretch vibrations. ${ }^{25}$ From the linear IR spectrum we deduced that the AOT used contains $1 \mathrm{H}_{2} \mathrm{O}$ molecule/25 AOT molecules, which was taken into account in determining the appropriate amounts of the constituents. The water mass 
fraction of all samples was 0.015. Pentane and iso-octane were added in a 1:1 volume ratio. Samples were prepared by mixing the constituents without further purification. The micellar solution is contained within a copper sample cell consisting of two $2 \mathrm{~mm}$ thick sapphire windows that are separated by a $500 \mu \mathrm{m}$ thick Kalrez spacer. The sample is mounted within a closed cycle cryostat (CTI-Cryogenics).

The micellar solutions were frozen rapidly by plunging the sample holder into liquid nitrogen, with resulting cooling rates on the order of $10^{2} \mathrm{~K} / \mathrm{s} .{ }^{26,27}$ Even faster cooling rates $\left(>10^{5} \mathrm{~K} / \mathrm{s}\right)$ can be reached when freezing isolated micrometer sized droplets. In such cases water can be frozen into other forms than the usual hexagonal ice, such as amorphous or cubic ice. ${ }^{14,28}$ Based on cooling speed alone, such different forms of ice are not expected to form in reverse micelles, as they are contained in bulk solutions that freeze relatively slowly.

We used a 1:1 ratio of iso-octane and pentane as apolar solvent, since we found this mixture to form a clear glass upon freezing. After freezing the sample the cryostat is closed and vacuumed. The temperature is subsequently raised to $180 \mathrm{~K}$, so that ice deposited from the air onto the sample cell can be pumped off. At $180 \mathrm{~K}$ the apolar glass matrix melts and leaves the frozen micelles suspended.

The stability of reverse micelles is known to depend on temperature, and at low temperatures spontaneous solution demixing can occur. ${ }^{21}$ Reverse micelles will then shed water from their interior, ${ }^{19,22}$ thereby strongly decreasing the micelle water content. The rate of water shedding depends on temperature and micelle size but has been reported to be a fairly slow process taking up to minutes. ${ }^{20}$ During water shedding, phase separation takes place in which the micellar structures break down and visual inspection reveals that large ice clusters form that sink to the bottom of the sample cell.

We use fast cooling to prevent water shedding from the micelle interior. In this way we prepare samples that are optically clear, suggesting the inhibition of water shedding. The micellar stability of our samples is characterized in Sec. III A. For normal micelles it has been well established that micellar structure can be retained when freezing the micellar solutions quickly, ${ }^{29-31}$ and similar reports exist for quickly frozen reverse micelles. ${ }^{26,27}$

\section{B. Pump-probe experiment}

We performed ultrafast midinfrared pump-probe spectroscopy on the $\mathrm{O}-\mathrm{H}$ stretch vibration of diluted HDO in $\mathrm{D}_{2} \mathrm{O}$ at $180 \mathrm{~K}$. In the experiment, a first intense midinfrared light pulse excites the $\mathrm{OH}$ oscillators, inducing transmission changes of a weak second time-delayed probe pulse. After excitation, the transmission is increased at frequencies matching the $v_{\mathrm{OH}}=0 \rightarrow 1$ transition (because of ground state depletion of the $\mathrm{OH}$-stretch vibration and stimulated emission out of the $v_{\mathrm{OH}}=1$ state), while the transmission is decreased at the more redshifted frequencies matching the $v_{\mathrm{OH}}=1 \rightarrow 2$ transition (because of absorption due to the induced population in the $v_{\mathrm{OH}}=1$ state).

The femtosecond midinfrared light source has been described previously elsewhere. ${ }^{11}$ The generated probe pulses

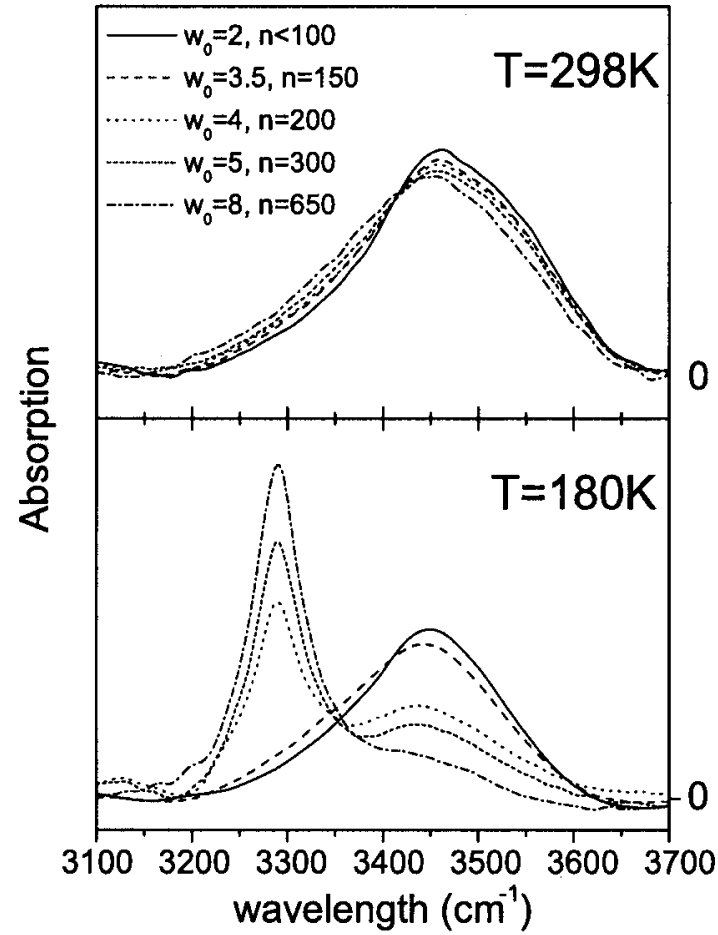

FIG. 1. Linear OH-stretch absorption for AOT reverse micellar solutions at room temperature (top) and at $180 \mathrm{~K}$ (bottom). The micelle size increases with the parameter $w_{0}=[$ water $] /[$ surfactant $] . n$ equals the approximate number of water molecules per reverse micelle (Refs. 23 and 24).

are spectrally broad compared to the pump pulses $\left[150 \mathrm{~cm}^{-1}\right.$ and $80 \mathrm{~cm}^{-1}$ full width at half maximum (FWHM), respectively], and the time resolution is $\sim 150 \mathrm{fs}$. After reflecting the probe beam off a glass window to lower its intensity by $95 \%$, the pump and probe beams are overlapped and focused at the sample inside the cryostat. The transmitted probe beam is sent through a polarizer and spectrally resolved on a nitrogen-cooled $\mathrm{HgCdTe}$ detector array using a polychromator with spectral resolution of $15 \mathrm{~cm}^{-1}$. Using an automated rotatable mount, the polarizer can be set either parallel or perpendicular with respect to the pump polarization, and at each delay point both probe components are detected consecutively. The sapphire windows introduce some birefringence, which causes a limited amount of mixing of the parallel and perpendicular components $(\sim 20 \%)$. We performed reference measurements using more fragile $\mathrm{CaF}_{2}$ windows that do not introduce any birefringence. These measurements confirmed that no anisotropic dynamics was present in the system. We chose to only use the perpendicular data for further analysis, which is the probe polarization least susceptible to scattered pump light.

\section{RESULTS AND DISCUSSION}

\section{A. Ultrafast heating dynamics and micelle stability}

Linear infrared absorption spectra for the $\mathrm{OH}$-stretch absorption of HDO: $\mathrm{D}_{2} \mathrm{O}$ in AOT micellar solutions are displayed in Fig. 1, both at room temperature (top) and at $180 \mathrm{~K}$ after fast freezing (bottom). For small reverse micelles $\left(w_{0}\right.$ $\leqslant 3.5)$ the $\mathrm{OH}$-stretch spectrum remains broad upon freezing, which shows that a large spread in hydrogen bond lengths is 


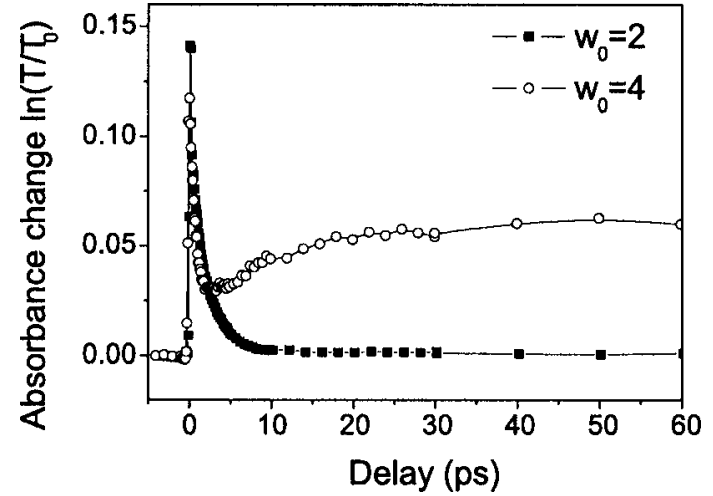

FIG. 2. Pump induced absorbance change plotted as a function of probe delay for a sample of $w_{0}=2$ and 4 . The pump/probe frequencies were $3450 / 3444 \mathrm{~cm}^{-1}$ and $3300 / 3293 \mathrm{~cm}^{-1}$, respectively. Much stronger absorbance changes related to heating are observed for samples of $w_{0} \geqslant 4$ than for $w_{0}<4$.

present at low temperatures. The width of the absorption agrees well with that recorded for amorphous $\mathrm{HDO}: \mathrm{D}_{2} \mathrm{O}$ ice. ${ }^{32}$ For micelles of $w_{0} \geqslant 4$ an additional narrow absorption peak at $3300 \mathrm{~cm}^{-1}$ appears, which becomes the dominant feature for large micelles. This peak appears at the same spectral position as the $\mathrm{OH}$-stretch absorption for bulk crystalline ice $\mathrm{HDO}: \mathrm{D}_{2} \mathrm{O}$.

Figure 2 shows the pump-induced transient absorption change as a function of delay for micelles with $w_{0}=2$ and $w_{0}=4$. Both pump and probe are tuned to the maximum of the absorption band. At delay zero the transmission is increased because of ground state depletion of the $\mathrm{OH}$-stretch vibration and stimulated emission out of the $v_{\mathrm{OH}}=1$ state. The transmission increase is followed by vibrational relaxation and thermalization dynamics. The vibrational relaxation will be discussed in detail in the next subsection. A striking difference between the signals measured for the two micelles is the magnitude of the signal at delays $>10$ ps. For a micelle with $w_{0}=2$ the signal has practically vanished, whereas for a micelle with $w_{0}=4$ a large transmission change related to thermalization dynamics is observed.

The signal measured at large delays reflects the amount of heating induced by the pump-pulse excitation. Heating affects the cross-section spectrum of the $\mathrm{OH}$-stretch vibration by a blueshift and decrease in amplitude. ${ }^{33}$ These crosssection changes result in absorption changes $\Delta \alpha_{T}(\omega, t)$ for a second time-delayed infrared pulse probing the sample. The amplitude of this absorption change is known to be linearly dependent on the amount of heat absorbed by the sample for both water $^{34}$ and ice. ${ }^{35}$ The amplitude of the absorption change can, thereby, act as a local thermometer.

Figure 3(a) shows the amplitude of the transmission changes induced by the heating effect at $60 \mathrm{ps}$ time delay. The amplitude of the heat effect is scaled to the amplitude of the transmission changes at $0.2 \mathrm{ps}$, to correct for the intensity of the excitation pulse (since the data for liquid micelles were recorded at magic angle instead of at a perpendicular probe polarization, ${ }^{11}$ the relative amplitude of the heating effect was upscaled for these measurements by a factor 5/3). For $w_{0} \leqslant 3.5$ the heating effect is equally small for frozen micellar solutions as for liquid micellar solutions. Directly

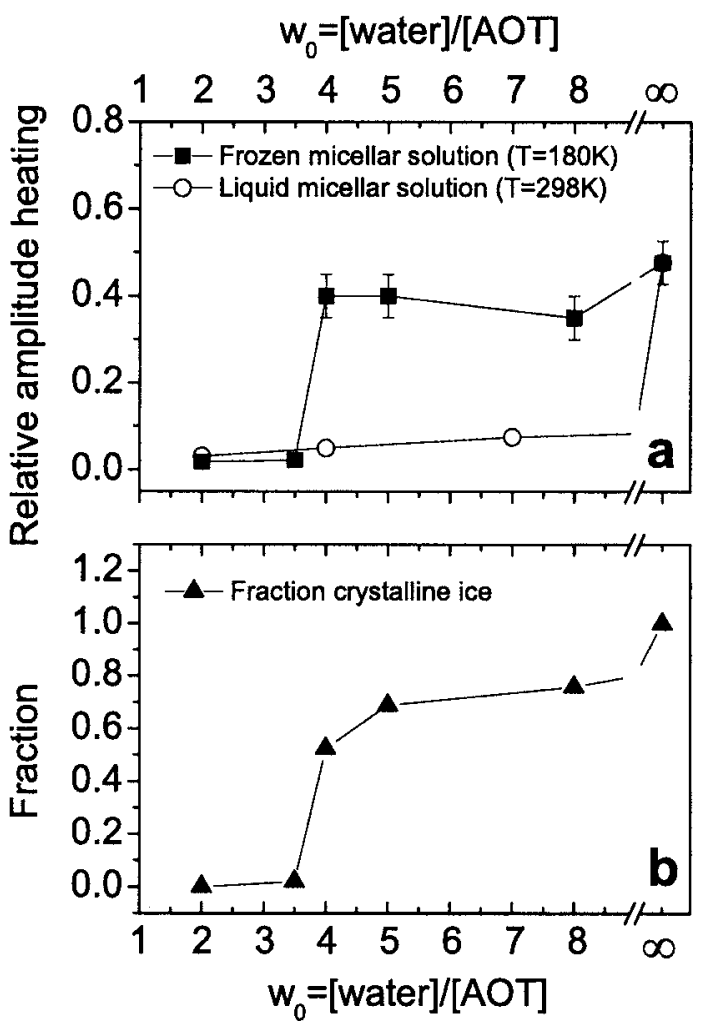

FIG. 3. (a) Amplitude of the measured absorption changes induced by heating at 60 ps probe delay (at the probe frequency of maximum absorption change). The amplitudes are scaled relative to the absorption change induced by population transfer at $0.2 \mathrm{ps}$, to divide out any dependence of the signal amplitude on the laser intensity. (b) Fraction crystalline ice as a function of the parameter $w_{0}$. This fraction is determined by fitting the linear absorption spectra of Fig. 1 to a linear combination of the $w_{0}=2$ and bulk crystalline ice spectra.

after vibrational relaxation of a liquid reverse micellar solution, the sample will contain hot reverse micelles suspended in a cooler apolar solvent. Because the heat is not homogeneously distributed within the sample, the micelles will cool to the surrounding solvent on a picosecond timescale. ${ }^{24}$ When the micelle cooling is complete, the heat is equilibrated over the micelles and the solvent. Since the solvent takes up the majority of the sample volume, the final heating effect on the micelles will be very small.

For frozen micellar solutions of $w_{0} \geqslant 4$ very slow cooling is observed, and more than $50 \%$ of the heating signal remains at $1000 \mathrm{ps}$. At $60 \mathrm{ps}$ the heating effect still has a similar amplitude as a heated sample of bulk ice of the same concentration of HDO molecules, while for liquid micellar solutions of the same $w_{0}$ at this delay time cooling is already complete. The heating dynamics reveal that our preparation method leads to stable micelles up to $w_{0}=3.5$ and that for $w_{0} \geqslant 4$ the micelles fall apart forming large clusters of ice. The cooling times of these clusters are slower than 1000 ps, which implies that the clusters must have a typical size larger than $10 \mathrm{~nm} .^{24}$ The fact that the solutions are optically clear puts an upper limit of $500 \mathrm{~nm}$ to the cluster size.

It is instructive to correlate the heating dynamics with the appearance of the crystalline ice peak in the linear absorption spectrum. For all micellar solutions the linear absorption spectrum can be fitted by a linear combination of 


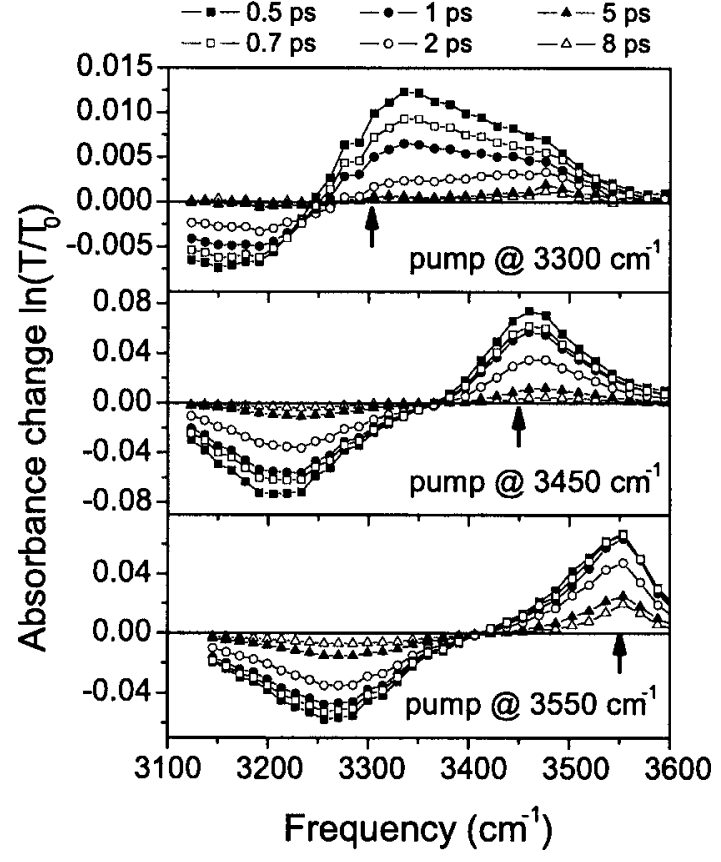

FIG. 4. Transient absorbance change for a micelle of $w_{0}=2$ at various probe delays, when exciting the sample by a pump pulse centered at $3300 \mathrm{~cm}^{-1}$ (top), $3450 \mathrm{~cm}^{-1}$ (middle), and $3550 \mathrm{~cm}^{-1}$ (bottom). The transient spectrum shifts with pump frequency.

the spectrum of the $w_{0}=2$ sample and that of bulk $\mathrm{HDO}: \mathrm{D}_{2} \mathrm{O}$ ice. Only the relative fraction of the two components varies over the range of $w_{0}$ considered. The fraction crystalline ice for each sample is plotted as a function of $w_{0}$ in Fig. 3(b). From this figure it is clear that in the transition from a micellar solution of $w_{0}=3.5$ to $w_{0}=4$ the relative amount of crystalline ice is sharply increasing. The transition takes place at the same value of $w_{0}$ where from the heating dynamics we identified the onset of micellar breakup [see Fig. 3(a)]. Most crystalline ice will therefore be found outside the reverse micelles, coalesced in larger ice crystals.

We conclude that the aqueous core of the micelles is stabilized in an amorphous form of ice for micellar structures up to $w_{0}=3.5$. This micelle contains approximately $150 \mathrm{wa}-$ ter molecules. ${ }^{23,24}$ Breakup of larger reverse micelles is observed to coincide with the appearance of bulk crystalline ice in the sample.

\section{B. Vibrational dynamics of micelle-confined amorphous ice}

Figure 4 shows the pump-induced transient absorption change plotted for different pump frequencies and varying probe delays for a micelle with $w_{0}=2$. After excitation by the pump, the transmission is increased at frequencies matching the $\mathrm{v}_{\mathrm{OH}}=0 \rightarrow 1$ transition (because of ground state depletion of the $\mathrm{OH}$-stretch vibration and stimulated emission out of the $v_{\mathrm{OH}}=1$ state), whereas the transmission is decreased at the more redshifted frequencies matching the $v_{\mathrm{OH}}=1 \rightarrow 2$ transition (where the induced population in the $v=1$ state causes absorption). The pump-probe spectra obtained with different pump frequencies are shifted in frequency and remain so at all delays. The amorphous ice apparently contains a strongly inhomogeneous distribution of $\mathrm{OH}$ oscillators that

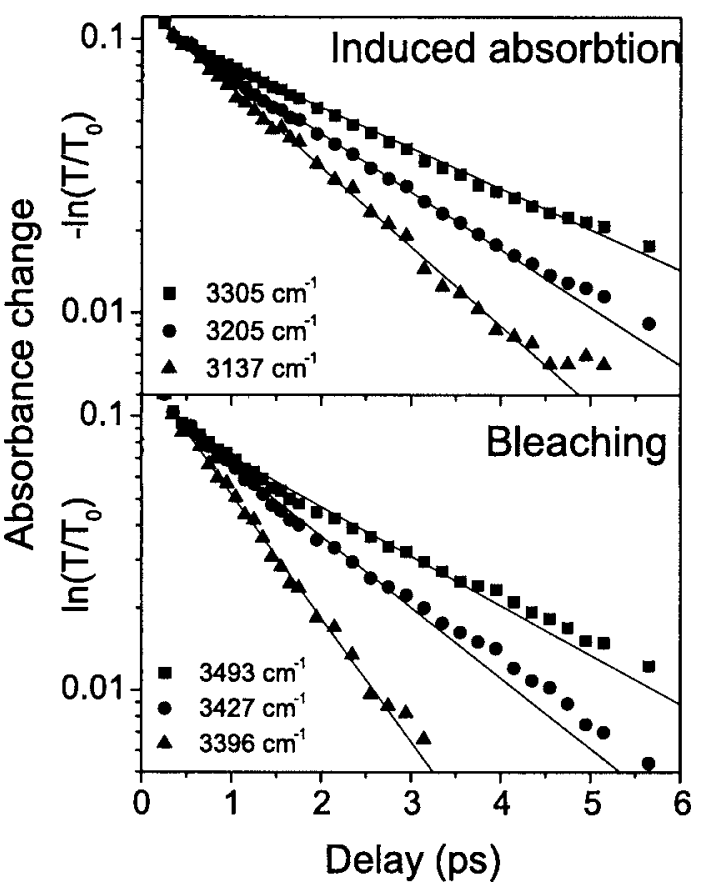

FIG. 5. Pump induced absorbance change plotted as a function of probe delay at various probe frequencies. Vibrational relaxation is observed to be strongly frequency dependent; the transient absorption change is well described by a monoexponential decay with a time constant depending on the probe frequency.

are spectrally different and do not interchange their absorption frequencies. The $\mathrm{OH}$-stretch frequency is strongly correlated to the length of the donated hydrogen bond. For this reason, the absence of any spectral dynamics shows the absence of large fluctuations in hydrogen bond lengths. The amorphous ice apparently contains a static ensemble of different hydrogen bonds.

It should be noted that a large amount of the amorphous ice will be surfactant bound water. The interaction between the AOT surfactant and water can be strong, and its sulfonate headgroup can accept hydrogen bonds from several water molecules. ${ }^{36}$ Therefore, the majority of the micellar amorphous ice may, in fact, be bound to surfactant molecules.

The vibrational relaxation of the micellar amorphous ice is observed to be very frequency dependent. This is illustrated in Fig. 5, where we show the transient absorption at different frequencies in time. The relaxation decay constant increases with absorption frequency from 0.7 to $4 \mathrm{ps}$. The vibrational relaxation of HDO water in liquid micelles has been previously described with a two-component model. ${ }^{11}$ Only two relaxation times were sufficient to describe the vibrational dynamics at all frequencies within the absorption band. The two components in the vibrational relaxation could be related to two different types of water molecules, bulklike molecules in the core of the micelles $\left(T_{1}\right.$ $=0.7-1 \mathrm{ps})$ versus interfacial molecules bound to the AOT surfactant molecules $\left(T_{1}=2.8 \mathrm{ps}\right)$. The vibrational relaxation presented here for frozen micelles cannot be described with two time constants, and a much more inhomogeneous distribution of relaxation times is observed.

While for bulk HDO: $\mathrm{D}_{2} \mathrm{O}$ water the vibrational relaxation becomes faster upon freezing ${ }^{37}$ (from 0.8 to $0.4 \mathrm{ps}$ ), for 


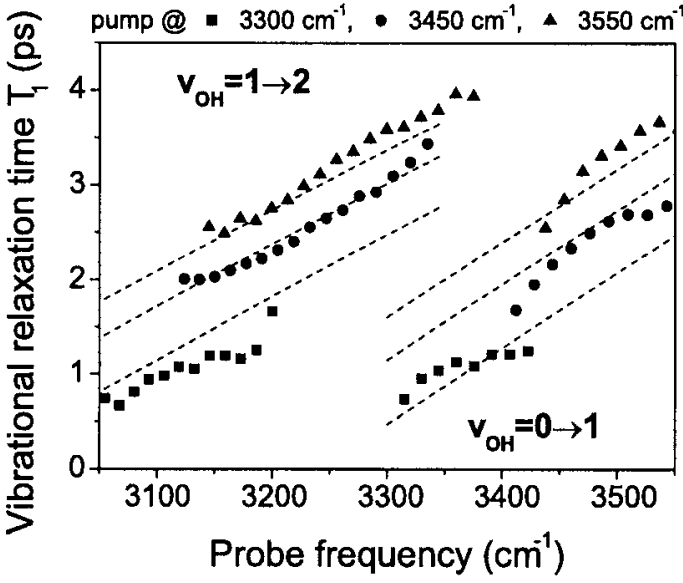

FIG. 6. Fitted exponential time constants for the transient absorption change recorded at different probe frequencies. The time-constants are observed to also depend on the center frequency of the pump (data shown for a pump centered at $3300 \mathrm{~cm}^{-1}, 3450 \mathrm{~cm}^{-1}$, or $3550 \mathrm{~cm}^{-1}$ ). Dotted lines are fits that assume a linear dependence of the vibrational relaxation time on the $\mathrm{OH}$ stretch frequency and a homogeneous linewidth of $55 \mathrm{~cm}^{-1}$.

micellar $\mathrm{HDO}: \mathrm{D}_{2} \mathrm{O}$ it becomes slower. The freezing of bulk water results in a strong narrowing and redshift of the $\mathrm{OH}$ stretch absorption, related to increased structural order and hydrogen bond strengthening. The redshift brings the $\mathrm{OH}-$ stretch energy in closer resonance with the HOD bending overtone, facilitating faster vibrational relaxation. The $\mathrm{OH}-$ stretch spectrum of amorphous micellar ice does not undergo such a redshift. Therefore, the energy gap with the overtone of the bending mode remains large and the relaxation involves lower-frequency modes to make up for the energy gap. At low temperatures the coupling to these modes will be reduced, because these modes will be less populated due to a small Boltzmann factor. This effect likely explains why the relaxation is even slower than for $\mathrm{HDO}: \mathrm{D}_{2} \mathrm{O}$ micellar water in the liquid phase.

The relaxation at each frequency can be well described by a single exponential decay (see Fig. 5). The fitted time constants are plotted in Fig. 6. Besides a dependence on the probe frequency, the vibrational relaxation also depends on the pump frequency. At a fixed probe frequency, more slowly relaxing molecules are observed if these are excited by a pump that has been tuned to the blue side of the spectrum. This can be understood by noting that the inhomogeneously broadened $\mathrm{OH}$-stretch absorption consists of an ensemble of homogeneous absorption lines with different center frequencies. At a fixed probe frequency, more high frequency homogeneous lines will be excited by a more blue pump frequency, which have a longer associated $T_{1}$ relaxation time.

We model the pump selectivity in the observed $T_{1}$ by assuming a frequency dependence for $T_{1}$ and a single homogeneous linewidth across the entire $\mathrm{OH}$-stretch absorption band. We define $S_{\text {hom }}\left(\omega, \omega^{\prime}\right)$ as a Gaussian homogeneous line of width $\sigma_{\text {hom }}$ and center frequency $\omega^{\prime}$. We can deconvolute the homogeneous broadening from the linear absorption spectrum to obtain the frequency distribution of homogeneous lines $I(\omega)$, such that the linear absorption spectrum equals $\int I\left(\omega^{\prime}\right) S_{\text {hom }}\left(\omega-\omega^{\prime}, 0\right) d \omega^{\prime}$. The observed $T_{1}$ at a probe frequency $\omega_{\mathrm{pr}}$ will then be the weighted average over all the homogenous distributions that have been excited by the pump centered at frequency $\omega_{\mathrm{pu}}$ :

$$
T_{1}\left(\omega_{\mathrm{pr}}, \omega_{\mathrm{pu}}\right)=\frac{\int_{0}^{\infty} d \omega S_{\mathrm{pu}}\left(\omega, \omega_{\mathrm{pu}}\right) S_{\mathrm{hom}}\left(\omega_{\mathrm{pr}}, \omega\right) I(\omega) \cdot T_{1}(\omega)}{\int_{0}^{\infty} d \omega S_{\mathrm{pu}}\left(\omega, \omega_{\mathrm{pu}}\right) S_{\mathrm{hom}}\left(\omega_{\mathrm{pr}}, \omega\right) I(\omega)} .
$$

$S_{\mathrm{pu}}\left(\omega, \omega_{\mathrm{pu}}\right)$ equals the pump spectrum when centered at $\omega_{\mathrm{pu}}$, which is similar to a Gaussian of FWHM $80 \mathrm{~cm}^{-1} . T_{1}(\omega)$ equals the relaxation time as a function of $\mathrm{OH}$-stretch absorption frequency. In view of the observed inhomogeneities in the vibrational relaxation, the homogeneous linewidth will be much smaller than the linear absorption spectrum. In this limit we can approximate $I(\omega)$ with the linear absorption spectrum itself.

We find a good description of the measured relaxation times of the $v_{\mathrm{OH}}=0 \rightarrow 1$ transition when $T_{1}(\omega)$ varies linearly with frequency according to $T_{1}(\omega)=A+B\left(\omega-\omega_{0}\right)$, where $\omega_{0}$ is the central frequency of the OH-stretch band $\left(\omega_{0}\right.$ $=3450 \mathrm{~cm}^{-1}$ ). For the $v_{\mathrm{OH}}=1 \rightarrow 2$ absorption the same relation holds, but since this transition is redshifted by the anharmonicity $\Delta$ and broadened by a factor $k=1.2$, we make the transformation $B \rightarrow B / k$ and $\omega_{0} \rightarrow \omega_{0}-\Delta$. By a least squares fit we find $A=2.4 \pm 0.2 \mathrm{ps}, \quad B$ $=0.014 \pm 0.002 \mathrm{ps} / \mathrm{cm}^{-1}, \quad \Delta=255 \pm 10 \mathrm{~cm}^{-1}, \quad$ and $\sigma_{\text {hom }}$ $=55 \pm 5 \mathrm{~cm}^{-1}$. The fitted curves are displayed as dotted lines in Fig. 6.

In view of the spectral width of the pump $\left(80 \mathrm{~cm}^{-1}\right)$ and the homogeneous linewidth $\left(55 \mathrm{~cm}^{-1}\right)$, a distribution of oscillators with different relaxation times $T_{1}$ will be excited at each probe frequency. This leads in principle to a slight multiexponential decay of the pump-probe signal. Oscillators with a longer $T_{1}$ will contribute longer to the signal than oscillators with a shorter $T_{1}$, causing the pump-probe signal to decay somewhat slower at later delay times. A close inspection of the decay curves of Fig. 5 indeed reveal some multiexponential behavior.

Comparing data and fit of Fig. 6 shows that a fully linear relation between $T_{1}$ and the $\mathrm{O}-\mathrm{H}$ stretch frequency assumed in our model is a slight oversimplification. At low frequencies the $T_{1}$ increases with frequency somewhat more slowly than at high frequencies, an effect not included in our linear $T_{1}$-frequency relation. For many hydrogen-bonded systems $T_{1}$ is related to the $\mathrm{O}-\mathrm{H}$ stretch frequency not according to a linear dependence but by a nearly inverse quadratic relation: $T_{1}(\omega)=a\left(\omega_{\mathrm{OH}}-\omega_{\mathrm{OH}, g}\right)^{-1.8}$, with $a$ a constant and $\omega_{\mathrm{OH}, g}$ the vibrational frequency of the $\mathrm{O}-\mathrm{H}$ group in the gas phase. ${ }^{38,39}$ This relation shows a stronger frequency dependence of the $T_{1}$ at blue frequencies compared to red frequencies, but this nonlinearity was found to be far too strong to be in accordance with our experimental data. The formula was derived for the case of vibrational predissociation, in which vibrational energy can be transferred to the $\mathrm{O} \cdots \mathrm{O}$ hydrogen bond stretch coordinate only. In amorphous ice, the relaxation likely involves energy transfer to the overtone of the bending mode. This prevents the relaxation time to go to infinity near 
the gas-phase frequency, as predicted by the inverse quadratic equation. A linear frequency dependence is more adequate in describing the experiment.

The $v_{\mathrm{OH}}=1 \rightarrow 2$ lineshape of the amorphous ice is slightly broader than the $v_{\mathrm{OH}}=0 \rightarrow 1$ transition, by a factor of 1.2. This strongly contrasts with the case of bulk HDO: $\mathrm{D}_{2} \mathrm{O}$ ice, ${ }^{40}$ where the $v_{\mathrm{OH}}=1 \rightarrow 2$ transition is broadened by a factor of 5 . The broadening in bulk ice is related primarily to a very short vibrational lifetime of the $v_{\mathrm{OH}}=2$ state. ${ }^{33}$ The $v_{\mathrm{OH}}=2$ state of bulk ice is resonant with the HDO bending overtone, which facilitates very fast vibrational energy relaxation. The amorphous ice studied here has a $\mathrm{OH}$-stretch spectrum that absorbs at more blueshifted frequencies, frustrating enhanced relaxation by this Fermi resonance. The $v_{\mathrm{OH}}=1$ $\rightarrow 2$ transition is therefore not lifetime broadened.

The micellar amorphous ice that is hydrogen bonded to the sulfonate headgroup of the surfactant likely experiences a somewhat different $\mathrm{OH} \cdots \mathrm{O}$ potential than bulk water and ice. For liquid water a second broadening mechanism of the $v_{\mathrm{OH}}=1 \rightarrow 2$ transition was identified, related to proton delocalization in the $v_{\mathrm{OH}}=2$ state. ${ }^{33,41}$ In bulk ice this mechanism cannot be present as it does not agree with the narrow spread in hydrogen bond lengths. ${ }^{33}$ In the micellar amorphous ice the spread in hydrogen bond lengths is much broader; however, no $v_{\mathrm{OH}}=1 \rightarrow 2$ broadening is observed in this case as well. This confirms that the $\mathrm{OH} \cdots \mathrm{O}$ potential for amorphous ice contained in the micelles differs from that of liquid water.

\section{CONCLUSIONS}

We studied the vibrational dynamics of frozen water contained in AOT reverse micelles with femtosecond midinfrared pump-probe spectroscopy. From the thermalization dynamics we deduce that fast freezing of AOT reverse micellar solutions leaves the micellar structure intact up to a micelle water content of $w_{0}=3.5$. Larger micelles break up to form larger ice structures in the size range of $10-500 \mathrm{~nm}$. This micelle breakup is associated with the formation of crystalline ice in the micellar solution. Freezing micellar solutions at higher cooling rates is necessary to extend the stability range to micelles with a higher water content.

The intact reverse micelles of water content $w_{0} \leqslant 3.5$ contain amorphous ice, which may for a large part be hydrogen bonded to AOT surfactant molecules. The linear absorption spectrum of this micellar amorphous ice is equally broad as for water in the liquid phase, which implies the existence of a large degree of structural disorder and spread in hydrogen bond lengths.

The vibrational relaxation rate of micellar amorphous ice is found to increase almost linearly with the $\mathrm{OH}$-stretch absorption frequency, from 0.7 to $4 \mathrm{ps}$. Compared to the relaxation rate of micellar water in the liquid phase ${ }^{11}$ (surfactant bound $2.8 \mathrm{ps}, \mathrm{D}_{2} \mathrm{O}$ bound $0.7-1 \mathrm{ps}$ ), the average vibrational relaxation of the amorphous water slows down upon freezing. This contrasts with the case of bulk $\mathrm{HDO}: \mathrm{D}_{2} \mathrm{O}$, for which the vibrational relaxation rate increases (from $740 \mathrm{fs}$ at $T=298 \mathrm{~K}$ to $420 \mathrm{fs}$ at $T=180 \mathrm{~K}){ }^{37}$ From the pumpfrequency dependence of the vibrational relaxation, we deduce that the homogeneous linewidth equals $55 \pm 5 \mathrm{~cm}^{-1}$.
The linewidth of the $v_{\mathrm{OH}}=1 \rightarrow 2$ transition of the micellar amorphous ice is only slightly broadened compared to the $v_{\mathrm{OH}}=0 \rightarrow 1$ transition. This differs from the case of liquid $\mathrm{HDO}: \mathrm{D}_{2} \mathrm{O}$, which has equal structural disorder, but where the $v_{\mathrm{OH}}=1 \rightarrow 2$ is broadened significantly due to proton delocalization in the $v_{\mathrm{OH}}=2$ state. $^{41}$ The $\mathrm{OH}$-stretch potential of the amorphous ice must thus be different from that of bulk water.

\section{ACKNOWLEDGMENTS}

This work is part of the research program of the Stichting voor Fundamenteel Onderzoek der Materie (FOM), which is financially supported by the Nederlandse Organisatie voor Wetenschappelijk Onderzoek (NWO). We thank Mischa Bonn for critically reading the manuscript. Hinco Schoenmaker is gratefully acknowledged for technical support.

${ }^{1}$ N. E. Levinger, Science 298, 1722 (2002).

${ }^{2}$ M. Wong, J. K. Thomas, and T. Nowak, J. Am. Chem. Soc. 99, 4730 (1977).

${ }^{3}$ M.-C. Belissent-Funel, S. H. Chen, and J.-M. Zanotti, Phys. Rev. E 51, 4558 (1995)

${ }^{4}$ J. Faeder and B. M. Ladanyi, J. Phys. Chem. B 194, 1033 (2000).

${ }^{5}$ A. Scodinu and J. T. Fourkas, J. Phys. Chem. B 106, 10292 (2002).

${ }^{6}$ G. M. Sando, K. Dahl, and J. C. Owrutsky, J. Phys. Chem. A 108, 11209 (2004).

${ }^{7}$ M. R. Harpham, B. M. Ladanyi, and N. E. Levinger, J. Chem. Phys. 121, 7855 (2004).

${ }^{8}$ A. M. Dokter, S. Woutersen, and H. J. Bakker, Phys. Rev. Lett. 94, 178301 (2005).

${ }^{9}$ H.-S. Tan, I. R. Piletic, and M. Fayer, J. Chem. Phys. 122, 174501 (2005).

${ }^{10}$ D. Cringus, J. Lindner, M. T. W. Milder, M. S. Pshenichnikov, P. Voehringer, and D. A. Wiersma, Chem. Phys. Lett. 408, 162 (2005).

${ }^{11}$ A. M. Dokter, S. Woutersen, and H. J. Bakker, Proc. Natl. Acad. Sci. U.S.A. 103, 15355 (2006).

${ }^{12}$ I. R. Piletic, D. E. Moilanen, D. B. Spry, N. E. Levinger, and M. Fayer, J. Phys. Chem. A 110, 4985 (2006).

${ }^{13}$ A. M. Dokter, S. Woutersen, and H. J. Bakker, J. Chem. Phys. 126, 124507 (2007).

${ }^{14}$ G. P. Johari, J. Chem. Phys. 122, 194504 (2005).

${ }^{15}$ J. Dore, Chem. Phys. 258, 327 (2000).

${ }^{16}$ P. Douzou, E. Keh, and C. Balny, Proc. Natl. Acad. Sci. U.S.A. 76, 681 (1979).

${ }^{17}$ C. R. Babu, V. J. Hilser, and A. J. Wand, Nat. Struct. Mol. Biol. 11, 352 (2004).

${ }^{18}$ N. V. Nucci and J. M. Vanderkooi, J. Phys. Chem. B 109, 18301 (2005).

${ }^{19}$ C. A. Munson, G. A. Baker, S. N. Baker, and F. V. Bright, Langmuir 20, 1551 (2004).

${ }^{20}$ A. K. Simorellis, W. D. V. Horn, and P. F. Flynn, J. Am. Chem. Soc. 128, 5082 (2006).

${ }^{21}$ M. Zulauf and H.-F. Eicke, J. Phys. Chem. 83, 480 (1979).

${ }^{22}$ W. D. V. Horn, A. K. Simorellis, and P. F. Flynn, J. Am. Chem. Soc. 127, 13553 (2005).

${ }^{23}$ S. Abel, F. Sterpone, S. Bandyopadhyay, and M. Marchi, J. Phys. Chem. B 108, 19458 (2004).

${ }^{24}$ G. Seifert, T. Patzlaff, and H. Graener, Phys. Rev. Lett. 88, 147402 (2002).

${ }^{25}$ S. Woutersen and H. J. Bakker, Nature (London) 402, 507 (1999).

${ }^{26}$ P. Baglioni, H. Nakamura, and L. Kevan, J. Phys. Chem. 95, 3856 (1991).

${ }^{27}$ W. Jahn and R. Strey, J. Phys. Chem. 92, 2294 (1988).

${ }^{28}$ G. Johari and O. Andersson, Theor. Chem. Acc. 461, 14 (2007).

${ }^{29}$ P. Baglioni and L. Kevan, J. Phys. Chem. 91, 1516 (1987).

${ }^{30}$ J. R. Bellare, T. Kaneko, and D. F. Evans, Langmuir 4, 1066 (1988).

${ }^{31}$ C. Lange and T. Wolff, Colloid Polym. Sci. 284, 214 (2005).

${ }^{32}$ M. Wojcik and V. Buch, J. Chem. Phys. 99, 2332 (1993). 
${ }^{33}$ A. M. Dokter and H. J. Bakker, J. Chem. Phys. 128, 024502 (2008).

${ }^{34}$ T. Steinel, J. B. Asbury, J. Zheng, and M. D. Fayer, J. Phys. Chem. A 108, 10957 (2004).

${ }^{35}$ H. Iglev, M. Schmeisser, K. Simeonidis, A. Thaller, and A. Laubereau, Nature (London) 439, 183 (2006).

${ }^{36}$ B. Derecskei, A. Derecskei-Kovacs, and Z. Schelly, Langmuir 15, 1981 (1999).
${ }^{37}$ S. Woutersen, U. Emmerichs, H. K. Nienhuys, and H. J. Bakker, Phys. Rev. Lett. 81, 1106 (1998).

${ }^{38}$ A. Staib and J. T. Hynes, Chem. Phys. Lett. 204, 197 (1993).

${ }^{39}$ R. E. Miller, Science 240, 447 (1988).

${ }^{40}$ G. Seifert, K. Weidlich, and H. Graener, Phys. Rev. B 56, 231 (1997).

${ }^{41}$ H. J. Bakker, H.-K. Nienhuys, G. Gallot, N. Lascoux, G. M. Gale, J.-C. Leicknam, and S. Bratos, J. Chem. Phys. 116, 2592 (2002). 